\section{References}

Brandt, R.I., Foley, W.J., Fink, G.H. \& Regan, W.J. (1970) Mechanism of perforation of the heart with production of hydropericardium by a venous catheter and its prevention. American Journal of Surgery, 119, 311.

Brown, C.A. \& Kent, A. (1956) Perforation of right ventricle by polyethylene catheter. Southern Medical Journal, 49, 466.

Greenhall, M.J., Blewitt, R.W. \& McMahon, M.J. (1975) Cardiac tamponade and central venous catheter. British Medical Journal, 2, 595.
Henzel, J.H. \& DeWeese, M.J. (1971) Morbid and mortal complications associated with prolonged central venous cannulation. Awareness, recognition and prevention. American Journal of Surgery, 121, 600.

Homesley, H.D. \& ZeleniK, J.S. (1971) Hazards of central venous pressure monitoring: pericardial tamponade, American Journal of Obstetrics and Gynecology, 109, 1216.

Johnson, C.E. (1966) Perforation of right atrium by a polyethylene catheter. Journal of the American Medical Association, 195, 176.

\title{
Pneumothorax and hydrothorax after subclavian vein cannulation
}

\author{
STEPHEN HolT \\ M.B., M.R.C.P.
}

\author{
ElizABeth Myerscough \\ M.B., Ch.B.
}

Sefton General Hospital, Liverpool L15 2HE

\begin{abstract}
Summary
A patient is described who developed a right pneumothorax and a left hydrothorax following percutaneous subclavian vein cannulation. The prevention, diagnosis and treatment of this complication are discussed.

\section{Introduction}

Intravenous nutrition through an indwelling venous catheter is an accepted procedure. Large vein catheters provide a stable route of administration for prolonged parenteral therapy. Several approaches for central venous cannulation have been used including the antecubital, internal and external jugular, femoral and subclavian veins, but no method is free of complication (Thomas, Carter and Lowder, 1969). The subclavian route has gained popularity because of easy access, a large venous flow and a reliable patency. Catheter stability and tolerance is good and infection is not generally a problem. Pneumothorax is a well documented complication of subclavian vein cannulation (Walker and Sanders, 1969). Other infrequent hazards include hydrothorax, haemothorax, massive subcutaneous emphysema, arteriovenous fistula, brachial plexus injury and air embolism (Latimer, 1971). The occurrence of two of these complications in one patient has not been previously reported.
\end{abstract}

Correspondence: Dr S. Holt, Liverpool Regional Cardiac Centre, Sefton General Hospital, Smithdown Road, Liverpool L15 $2 \mathrm{HE}$.

\section{Case report}

A 64-year-old female with severe endogenous depression had become dehydrated after a prolonged period of self-starvation and refusal to drink. The subclavian vein was cannulated using a percutaneous supraclavicular approach for the administration of hyperosmolar parenteral alimentation.

\section{Technique}

A no. 19 Seldinger needle $7.7 \mathrm{~cm}$ long, which is made up of an external cannula and an internal section containing a stylet with a bevelled point, was used. The needle was introduced into the right supraclavicular fossa at the clavisternomastoid angle but penetration of the right subclavian vein was not achieved. The procedure was repeated on the left side and the left subclavian vein was entered with ease. After removal of the central needle and stylet, blood was aspirated freely into a syringe. A spring guide wire, $80 \mathrm{~cm}$ long, with an external diameter of $0.889 \mathrm{~mm}$, was passed through into the vein under $\mathrm{X}$-ray control. A compound percutaneous catheter introducer, with an 8.5 FG (French gauge) outer sheath fitting over a 6-FG catheter, was inserted over the guide wire after the removal of the Seldinger cannula. The wire and inner catheter were then removed and a non-radiopaque catheter ( 5 FG) was introduced into the vein and its position checked by X-ray screening after injection of sodium diatrizoate. The catheter introducer was withdrawn from 
the neck, pressure applied over the puncture site and the stability of the catheter checked during deep breathing and coughing. The indwelling catheter was then secured using two skin sutures, one at the incision and the second about $2 \mathrm{~cm}$ laterally. Blood was aspirated from the indwelling catheter in its final position.

\section{Progress}

Rehydration was then commenced through the central venous line with $0.9 \%$ sodium chloride, $5 \%$ dextrose, aminosol and soya oil emulsion, in rotation, at a rate of $500 \mathrm{ml}$ hourly. The patient's condition remained satisfactory until $48 \mathrm{hr}$ after the cannulation when she developed tachypnoea, cyanosis, tachycardia and hypotension. Examination revealed clinical signs of a left pleural effusion. $\mathrm{X}$-ray performed at this stage demonstrated a left pleural effusion and a right apical pneumothorax. The infusion was discontinued, the central venous line removed and a thoracocentesis performed in the left posterior, eighth intercostal space. One litre of milky, blood-stained fluid was aspirated. Repeat thoracocentesis performed 2 days later yielded a further $400 \mathrm{ml}$ of similar fluid. The patient's condition gradually improved over the ensuing 4 weeks and she has no residual respiratory disability.

\section{Discussion}

The latent interval of 2 days between cannulation and the development of respiratory embarrassment suggests that the indwelling venous catheter had become displaced some time after insertion. Furthermore, the position of the catheter was checked immediately after insertion both by injection of radiopaque medium and aspiration of blood. Rudge, Berwick and McCall (1973) indicated that damage to a vein wall may occur hours or days after cannulation. Shafiq (1975) reported extravasation of hypertonic fluids from a central venous catheter into the right hemithorax and postulated that leakage could have occurred owing to gradual ulceration of the vein wall by the catheter. The development of hydrothorax in the present patient was presumably a result of movement of the polyethylene catheter through the vein wall into the left pleural space some time after insertion. The position of the venous line can be regularly checked by frequent aspirations of the catheter with a $10 \mathrm{ml}$ syringe. A free flow of blood back along the catheter into the syringe indicates an intravascular position. The authors consider that frequent demonstration of the correct position of the indwelling catheter is mandatory if this complication of infusion is to be avoided.

It seems likely that the pneumothorax in this patient occurred during the attempted cannulation of the right subclavian vein. The pneumothorax was presumably not recognized after screening of the patient with an image intensifier after venepuncture. It is suggested that subclavian venepuncture should be performed on the opposite side only after careful radiological screening, to exclude the occurrence of any complications which may have occurred during an earlier attempt at cannulation.

This report emphasizes the importance of radiological screening during and after subclavian vein cannulation and subsequent careful supervision of infusion via this route.

\section{Acknowledgment}

We wish to thank Dr E. J. Epstein for permission to report this patient.

\section{References}

LATIMER, R.D. (1971) Central venous catheterization. British Journal of Hospital Medicine, 5, 369.

Rudge, C.J., Berwick, M. \& MCColl, I. (1973) Hydrothorax after central venous catheterization. British Medical Journal, 3, 23.

ShafiQ, M. (1975) Extravasation of intravenous fluids in serous cavities. Journal of the Royal College of Surgeons of Edinburgh, 20, 208.

Thomas, C.S., Jr, Carter, J.W. \& Lowder, S.C. (1969) Pericardial tamponade from central venous catheters. Archives of Surgery, 98, 217.

WALKeR, M.M. \& SANDERS, R.C. (1969) Pneumothorax following supraclavicular subclavian venepuncture. Anaesthesia, 24, 453. 